\title{
A GRAPHIC ANALYSIS OF THE BIOMECHANICS OF THE MASSLESS BI-ARTICULAR CHAIN. APPLICATION TO THE PROXIMAL BI-ARTICULAR CHAIN OF THE HUMAN FINGER
}

\author{
J. N. A. L. Leijnse*† \\ *Department of Plastic and Reconstructive Surgery, †Department of Biomedical Physics and Technology, \\ Erasmus University, Rotterdam, The Netherlands
}

\begin{abstract}
In this paper a model is presented which visualises the biomechanical functioning of the loaded and unloaded theree-tendon bi-articular chain. This model allows to graphically determine in any position of the chain (i) the exact ranges of loads which can be sustained by the different motors; (ii) the motors forces; (iii) the feasibility of the (unloaded) equilibrium; and (iv) the conditions for the good controllability of the bi-articular chain. These results are applied to the proximal three-motor bi-articular chain of the human finger, when controlled by the superficial flexor, interosseus, and extensor only. It is shown that (i) the anatomic position of the superficial fiexor and extensor is a prime determinant in the good functioning of this chain; (ii) the proximal bi-articular chain of the human finger can well sustain certain flexion loads but is structurally weak for extension loads; and (iii) the chain is not optimally controllable.
\end{abstract}

\section{NOMENCLATURE}

MCP, PIP metacarpophalangeal joint, proximal interphalangeal joint of the finger

$F \quad$ (force in the) flexor

$E \quad$ (force in the) extensor

I (force in the) interosseus

$\Theta_{j} \quad$ angle of joint $j(j=1,2$ for MCP and PIP, respectively)

$\begin{array}{ll}r_{M j} & \text { moment arm of motor } M \text { at joint } j \\ v_{K j} & \text { moment arm of load vector } \mathbf{K} \text { at joint } j\end{array}$

$\mathbf{R}_{M}$ moment arm vector of motor $M$, with Cartesian

$V_{L_{1}} \quad$ coordinates $\left(r_{M 1}, r_{M 2}\right)$

$\mathbf{V}_{\mathbf{K}} \quad$ moment arm vector load $\mathbf{K}$, with Cartesian coor-

diantes $\left(v_{\mathrm{K} 1}, v_{K 2}\right)$

$K \cdot \mathbf{u}_{K} \quad$ modulus and unit vector of load $K=K \cdot u_{K}$

$\mathbf{u}_{F}, \mathbf{u}_{E}, \mathbf{u}_{I} \quad$ unit load vectors of the flexor, extensor and in terossues, respectively

$L_{1}, L_{2} \quad$ length of first and second phalanx

$\phi \quad$ angle of the distal phalanx and the load vector $\mathbf{u}_{K}$

Vector notations and expressions

(a) The scalar value representing the magnitude and direction (positive or negative) of the vector product $\mathbf{R}_{i} \times \mathbf{R}_{j}$ is indicated between brackets:

$$
\left[\mathbf{R}_{i} \times \mathbf{R}_{j}\right]=r_{i 1} \cdot r_{j 2}-r_{i 2} \cdot r_{j 1} \cdot
$$

(b) A 'non-negative sum' of two vectors $a_{1} \cdot \mathbf{R}_{1}+a_{2} \cdot \mathbf{R}_{2}$ is with $a_{1}, a_{2} \geqslant 0$; 'positive sum' is with $a_{1}, a_{2}>0$.

\section{INTRODUCTION}

The functioning of motors in a bi- or multi-articular chain is difficult to analyse because the motors simultaneously affect each joint they span. In 1955, Landsmeer provided a massless and frictionless bi-articular model to explain the control of the unloaded proximal bi-articular

Received in final form 11 Jamuary 1995. three-tendon chain of the human finger-later mathematically reformulated by Spoor and Landsmeer (1976), and further discussed in Leijnse and Kalker (1995). The Landsmeer model considers as the basis of control of the bi-articular chain the combined actions of two motors (the extensor and the flexor, clinically called 'extrinsic' motors), which cause the chain to collapse irreversibly. A third motor able to reverse this collapse is sufficient for complete control of the chain. The Landsmeer model provides a useful theoretical explanation of clinical phenomena, such as the claw-hand and the swan-neck deformity. However, it also presents some conceptual and practical limitations. Conceptually, it describes bi-articular control as the balance of the actions of a motor pair (the extrinsics) with respect to a single third motor (the interosseus, clinically called an 'intrinsic' motor), i.e. the 'extrinsic-intrinsic balance'. However, a comprehensive control model should be symmetric, in the sense that where it is possible to distinguish an 'intrinsic-extrinsic' balance, for the same reasons an extensor/interosseus-flexor balance, or a flexor/interosseus-extensor balance, or a mere three-tendon balance may also be distinguished. Moreover, controllable bi-articular chains without 'Landsmeer extrinsics' exist, i.e. in which no two tendons cross two joints at opposite sides. In that case the model cannot be applied. Another limitation is that the model only describes the functioning of the unloaded chain. More practically, the Landsmeer model is a kinematic (displacement) model, which implies that while its functioning is well demonstrable in physically moving models, it cannot be well visualised in a textbook, where the formulation of the results in mathematical expressions makes their appreciation by the clinician difficult.

The present paper attempts to remedy the limitations outlined above, and presents a model which (i) covers both the loaded and unloaded bi-articular chain, (ii) is 
completely graphically representable, and (iii) describes motor functioning in a symmetric way, i.e. without any assumption about the position of tendons. The model is based on the static torque equilibrium equations of the two joints. For the unloaded chain, the torque equations and the Landsmeer displacement model contain the same parameters (the moment arms of the motors at the different joints) and are therefore equivalent, as discussed in Spoor and Landsmeer (1976). The force model, however, also includes loaded situations. Moreover, it can be graphically analysed from two two-dimensional vector diagrams. These diagrams are obtained by associating with each motor or load two two-dimensional vectors (the moment arm vector and the load vector) which together completely describe the effects of the motor or load. Since vectors are abstract entities, this representation effectively reveals the symmetry in the functioning of the motors. The diagrams are: (i) the moment arm vector diagram, which allows determination of the motor forces and the feasibility of equilibrium, and (ii) the "load vector diagram', from which the loads which can be balanced by any two motors can be obtained as a function of the finger position. The diagrams are further used to define the general conditions on the loadability and the controllability of bi-articular chains. As an illustration. these conditions are investigated in the proximal bi-articular chain of the finger, when controlled by three motors: the interosseus, the extensor and the superficial flexor. For the reader with a theoretical interest the present model is discussed with respect to the results of Landsmeer (1955) and Spoor and Landsmeer (1976) in Appendix C.

\section{THE MODEL}

The torque equilibrium equations of the bi-articular three-tendon chain of Fig. 1(a) are:

$$
\begin{gathered}
E \cdot\left[\begin{array}{l}
r_{E 1} \\
r_{E 2}
\end{array}\right]+I \cdot\left[\begin{array}{l}
r_{I 1} \\
r_{12}
\end{array}\right]+F \cdot\left[\begin{array}{l}
r_{F 1} \\
r_{F 2}
\end{array}\right]=-K \cdot\left[\begin{array}{l}
v_{K 1} \\
v_{K 2}
\end{array}\right], \\
\infty>F, F, I, K \geqslant 0 .
\end{gathered}
$$

The motors are the extensor $(E)$, the flexor $(F)$ and the interosseus $(I)$. The motors and their forces are denoted by the same letter; and meaning follows from the context. The moment arms of a motor $M$ are denoted as $r_{M i}$, those

(a)
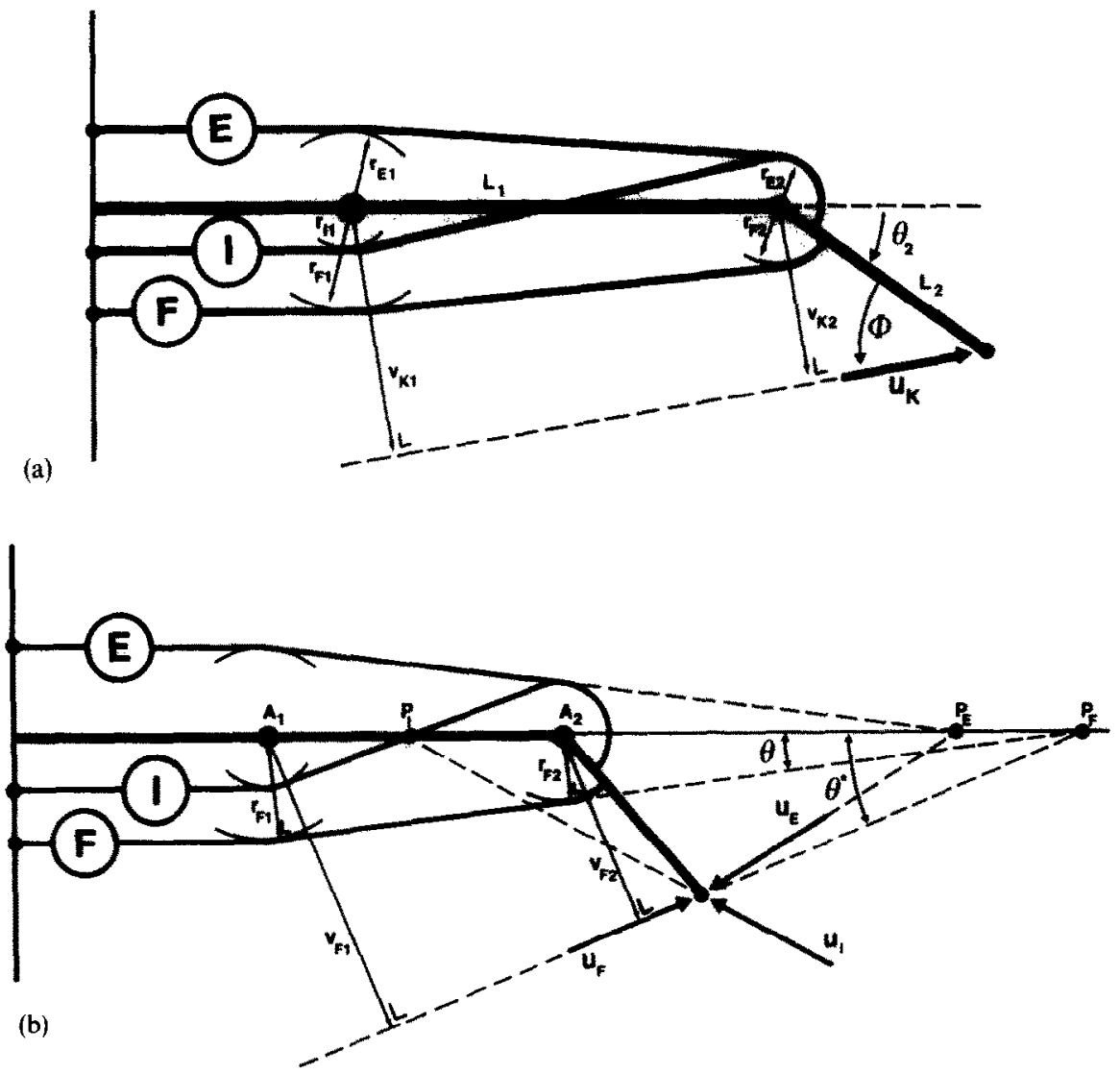

Fig. 1. (a) Bi-articular chain with three motors and unit load vector $\mathbf{u}_{\mathrm{K}}$. (b) Graphic construction of the load vector diagram. Three lines intersect at the points $P_{i}$ : (i) the line tangential to the circles centred at the joint axes, and with radii equal to the moment arms of the considered motor at these joints, (ii) the line $A_{1}-A_{2}$ connecting both joints axes, and (iii) the load line of the load vector $u_{\mathrm{x}}$. 
of the load $\mathbf{K}$ as $v_{K i}$. They are positive when the motor or the load induces an extension torque in a joint, otherwise (flexion torque) they are negative.

\section{The moment arm vector diagram}

The moment arms of a motor or load can be considered as the Cartesian coordinates of a vector, further called the moment arm vector $\mathbf{R}_{M}\left(r_{M 1}, r_{M 2}\right)$ or $\mathbf{V}_{K}\left(v_{K 1}, v_{K 2}\right)$ of this motor or load. As such, expression (2) can be written as a vector equation:

$$
E \cdot \mathbf{R}_{E}+I \cdot \mathbf{R}_{I}+F \cdot \mathbf{R}_{F}=-K \cdot \mathbf{V}_{K}
$$

The forces $E, I, F, K$ are now non-negative scalars of vector multiplication. The moment arm vectors can be represented in a vector diagram, further called the moment arm vector diagram. This diagram allows us to graphically determine the motor forces from a force parallelogram. For the unloaded chain $(K=0)$, this parallelogram is obtained by expressing the negative of the moment arm vector of one motor (e.g. the flexor $F$ ) as a non-negative sum of the moment arm vectors of the other motors [Fig. 2(a)]:

$$
E \cdot \mathbf{R}_{E}+I \cdot \mathbf{R}_{I}=-F \cdot \mathbf{R}_{F} .
$$

The forces $E$ and $I$ are then graphically obtained from the decomposition of the vector $-\mathbf{R}_{F}$ on the vectors $\mathbf{R}_{E}$ and $\mathbf{R}_{\boldsymbol{I}}$ :

$$
\frac{E}{F}=\frac{\left\|p_{\mathbf{R}_{E}}^{\mathbf{R}_{i}}\left(-\mathbf{R}_{F}\right)\right\|}{\left\|\mathbf{R}_{E}\right\|}, \quad \frac{I}{F}=\frac{\left\|p_{\mathbf{R}_{r}}^{\mathbf{R}_{\varepsilon}}\left(-\mathbf{R}_{F}\right)\right\|}{\left\|\mathbf{R}_{I}\right\|}
$$

[in which e.g. $p_{\mathbf{R}_{\varepsilon}^{\prime}}^{\mathbf{R}_{\prime}}\left(-\mathbf{R}_{F}\right)$ is the projection of $-\mathbf{R}_{F}$ on $\mathbf{R}_{E}$ along the direction of $\mathbf{R}_{I}$ ]. For the loaded chain, the moment arm vector $-V_{K}$ of the load is decomposed as a non-negative sum of the moment arm vectors of the motors [from expression (3)] [Fig. 2(b)]. Since the vector diagram is two-dimensional, any load moment arm vector $-\mathbf{V}_{\mathbf{K}}$ can be written as the sum of maximally two moment arm vectors, which means that any load in the bi-articular chain can be sustained by maximally two motors. When no motor moment arm vectors are collinear, the motor forces in the unloaded (a) and loaded (b) case can be written as (see Appendix A)

$$
\begin{gathered}
\frac{E}{F}=\frac{\left[\mathbf{R}_{I} \times \mathbf{R}_{F}\right]}{\left[\mathbf{R}_{E} \times \mathbf{R}_{I}\right]}, \\
\frac{I}{F}=\frac{\left[\mathbf{R}_{F} \times \mathbf{R}_{E}\right]}{\left[\mathbf{R}_{E} \times \mathbf{R}_{I}\right]}, \\
\frac{M_{i}}{K}=\frac{\left[\mathbf{R}_{j} \times \mathbf{V}_{K}\right]}{\left[\mathbf{R}_{i} \times \mathbf{R}_{j}\right]}, \\
\frac{M_{j}}{K}=\frac{\left[\mathbf{V}_{K} \times \mathbf{R}_{i}\right]}{\left[\mathbf{R}_{i} \times \mathbf{R}_{j}\right]},
\end{gathered}
$$

with $M_{i}$ and $M_{j}$ the motor pair balancing the load $\mathbf{K}$. These expressions illustrate that in this model mathematically no difference exists between a motor and a load with the same moment arms.

\section{The load vector diagram}

Presently explained is a diagram [Fig. 1(b)] which allows to graphically determine, in any position of the bi-articular chain, which motors balance a given load. For any motor $M$ in the bi-articular chain, a load vector $\mathbf{K}_{M}=K_{M} \cdot \mathbf{u}_{M}$ (with $\mathbf{u}_{M}$ a unit vector) exists which can be balanced by this motor alone, without the help of any other motor. The moment arms of this load vector can be determined from the generic expression (3), (set all motor forces except the generic motor $M$ to zero):

$$
\mathbf{V}_{M}=-\alpha_{M K} \cdot \mathbf{R}_{M}, \quad \alpha_{M K}=\frac{M}{K_{M}}>0 .
$$

Expression (7) states that the moment arms $v_{M i}$ of the load $\mathbf{u}_{M}$ must be proportional to the moment arms $r_{M i}$ of the motor $M$, and of opposite sign. The load vector $\mathbf{u}_{M}$ can be graphically constructed from a schematic drawing of the chain [Fig. 1(b)]. In this figure, the intersections of the lines tangent to the circles centred at the joint axes, with radii equal to the moment arms of the motors, and the line connecting both joint axes provide the points $P_{I}$, $P_{E}, P_{F}$. The load lines of the load vectors $\mathbf{u}_{I}, \mathbf{u}_{E}, \mathbf{u}_{F}$, of the motors $I, E, F$, respectively, are the lines connecting these points and the point of application of the load. This follows from the fact that (c.g. for the flexor $F$ )

$$
\begin{aligned}
& \left|r_{F j}\right|=\overline{A_{j} P_{F}} \sin \theta \quad(j=1,2), \\
& \left|v_{F j}\right|=\overline{A_{j} P_{F}} \sin \theta^{*}
\end{aligned}
$$

from which it follows that

$$
\frac{\left|v_{F 1}\right|}{\mid r_{F 1}}=\frac{\left|v_{F 2}\right|}{\left|r_{F 2}\right|}=\frac{\left|\sin \theta^{*}\right|}{|\sin \theta|}=\alpha_{F K},
$$

which defines the factor $\alpha_{M K}$ in expression (7) in geometric terms. Figure 1(b) shows that the direction of the load vector changes with the point of application and the joint positions. With extended second joint $\left(\theta_{2}=0\right)$, all load vectors are collinear with the line through the joint axes. This phenomenon is discussed further.

In the following, all loads which can be balanced by two motors alone are called the 'load range' of this motor pair. This load range consists of all loads which can be decomposed as a positive sum of the load vectors of the motor pair, according to the generic expression (in which for each motor pair the third motor is put to zero)

$$
K_{E} \cdot \mathbf{u}_{E}+K_{I} \cdot \mathbf{u}_{I}+K_{F} \cdot \mathbf{u}_{F}=K \cdot \mathbf{u}_{K}
$$

with $K_{E}, K_{I}, K_{F} \geqslant 0$. The load vector diagram thus straightforwardly provides the load ranges of the different motor pairs [Fig. 3(a)]. However, it does not allow determination of the exact motor forces. This is because the factors $K_{i}$ in expression (10) are only proportional (by the factors $\alpha_{M K}$ ) to the motor forces [expression (7)]. Moreover, the factors $\alpha_{M K}$ generally differ for each motor, as they depend on unrelated quantities (the moment arms of motors and load). However, these factors $\alpha_{M K}$ are positive, from which it follows that mathematically the motor forces $M$ and the decomposition factors $K_{M}$ in Fig. 3(a) have the same sign. This means that the load 
vector diagram and moment arm vector diagram are consistent. When in the moment arm vector diagram the negative moment arm vector $-V_{K}$ of a load $K$ is a positive sum of two motor moment arm vectors $\mathbf{R}_{i}, \mathbf{R}_{j}$, then in the load vector diagram the load $\mathbf{K}$ will also be a positive sum of the load vectors $u_{i}$ and $u_{j}$ of these motors $M_{i}$ and $M_{j}$, and vice versa.
Summary: the moment arm versus the load vector diagram

Together, the moment arm vector and load vector diagrams visualise all information enclosed in the force model (2).

(i) The moment arm vector diagram [expression (3)] allows to graphically determine the motor forces in the
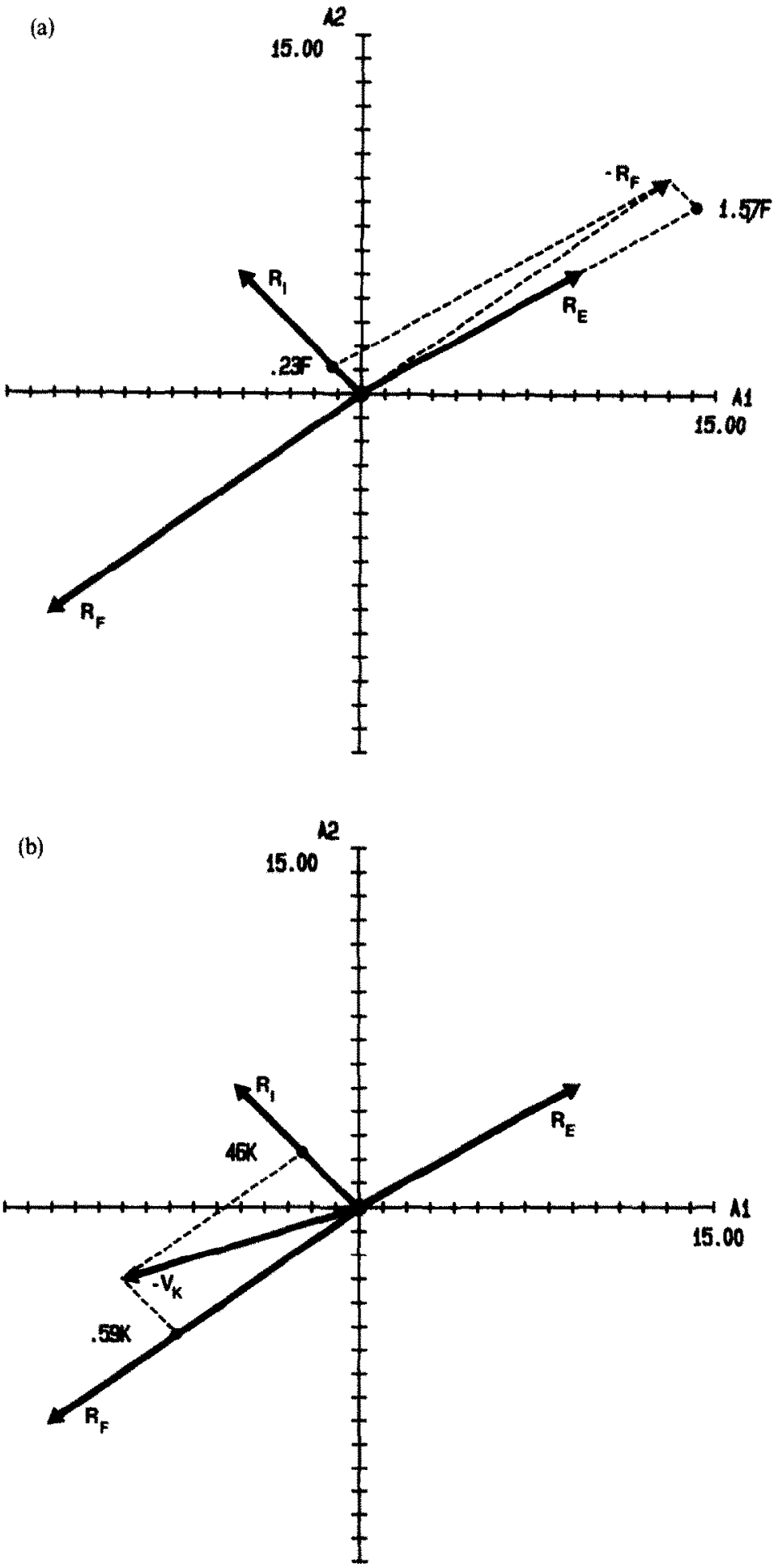

Fig. 2 (a,b) 

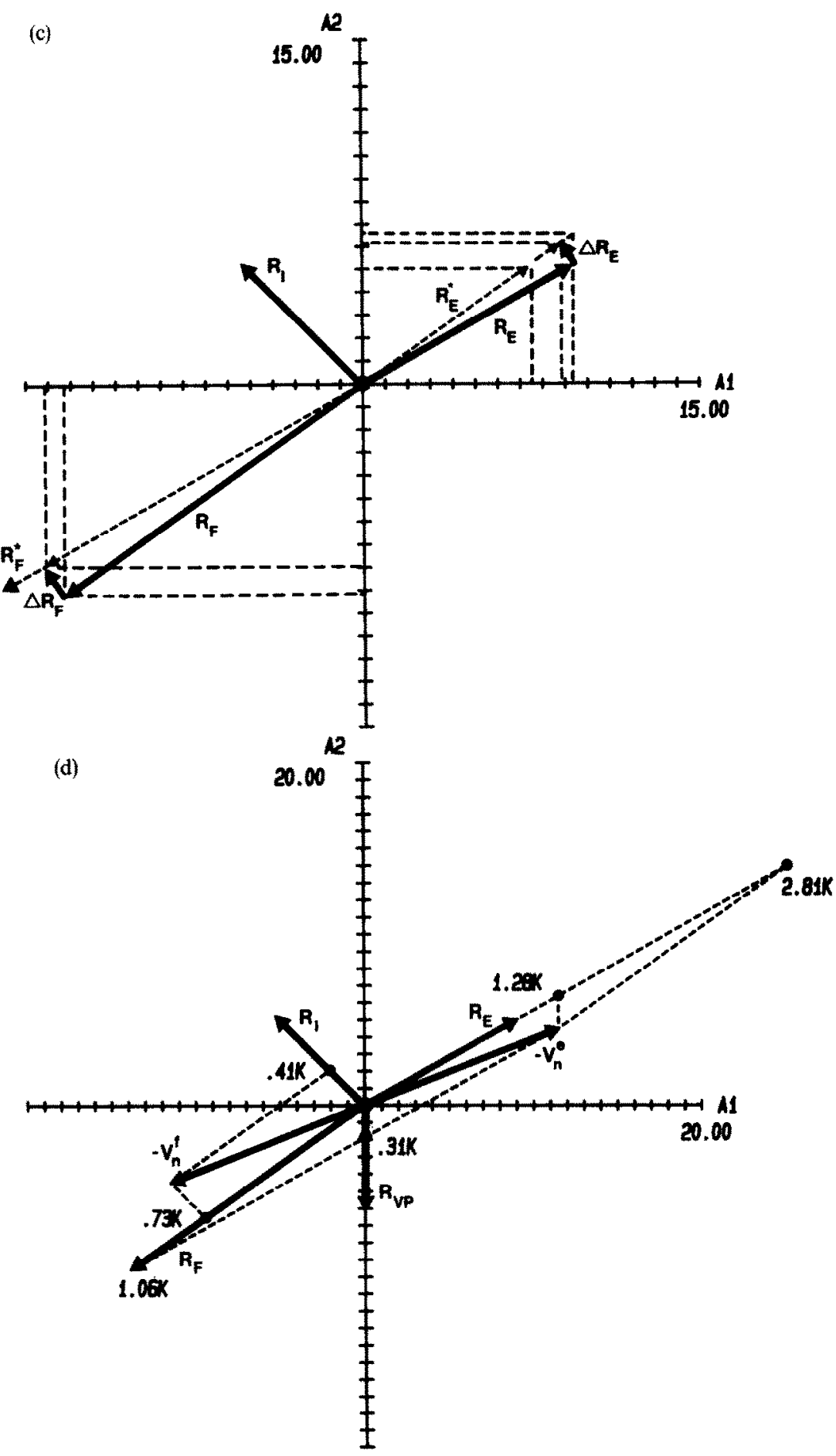

Fig. 2. (a) Moment arm vector diagram of the unloaded proximal bi-articular chain of the human finger. Moment arm values from Table 1. $A_{1}, A_{2}$; axes for the moment arms of the first and second joint. The motor forces follow from the force parallelogram: $F=F ; E / F=1.57 ; I / F=0.23$. (b) The motor forces with load $K$ as in Fig. 3(a). The moment arm vector $V_{K}$ of the load is scaled down by a factor 10 to fit in the picture. The motor forces are $I / K=4.6 ; F / K=5.9 ; E / K=0$. (c) Sensitivity analysis of the equilibrium of the proximal bi-articular chain of the human finger. When $\mathbf{R}_{F}$ or $\mathbf{R}_{E}$ are rotated so that they become collinear with $\mathbf{R}_{E}$ or $\mathbf{R}_{F}$, respectively (dashed vectors $\mathbf{R}_{F}^{*}$ or $\mathbf{R}_{E}^{*}$ ), the chain becomes uncontrollable. The dashed projection lines indicate the changes required. Changes of minimally $1.5 \mathrm{~mm}$ are required in one single moment arm to obtain an unstable chain, while these changes are somewhat greater for the moment arms of the first joint ( $A_{1}$ axis) than for the second joint ( $A_{2}$ axis). However, with simultaneous changes in the moment arms of the first and second joint, e.g. along the vectors $\Delta \mathbf{R}_{F}$ or $\Delta \mathbf{R}_{E}$, changes of less than 1 mm will unbalance the chain. (d) Motor forces in the extended proximal chain of the human finger loaded at the tip. $\mathbf{R}_{\mathrm{Vp}}$ : moment arm vector of the volar plate of the PIP joint. The moment arms of the extension and flexion load $\left(V_{n}^{e}, V_{n}^{t}\right)$ at the finger tip are scaled down by a factor 10 to fit in the picture. With slack volar plate the extension load (moment arm vector $-V_{n}^{*}$ ) is balanced by the flexor and extensor. Note the extremely large forces: $E / K=28.1 ; F / K=10.6$. With taut volar plate and relaxed flexor, the extensor force is more than halved: $E / K=12.8 ; V P / K=3.1$. A fexion load (moment arm vector $-V_{n}^{t}$ ) is balanced by the flexor and the interosseus with the forces: $F / K=7.3 ; I / K=4.1$. 
(a)

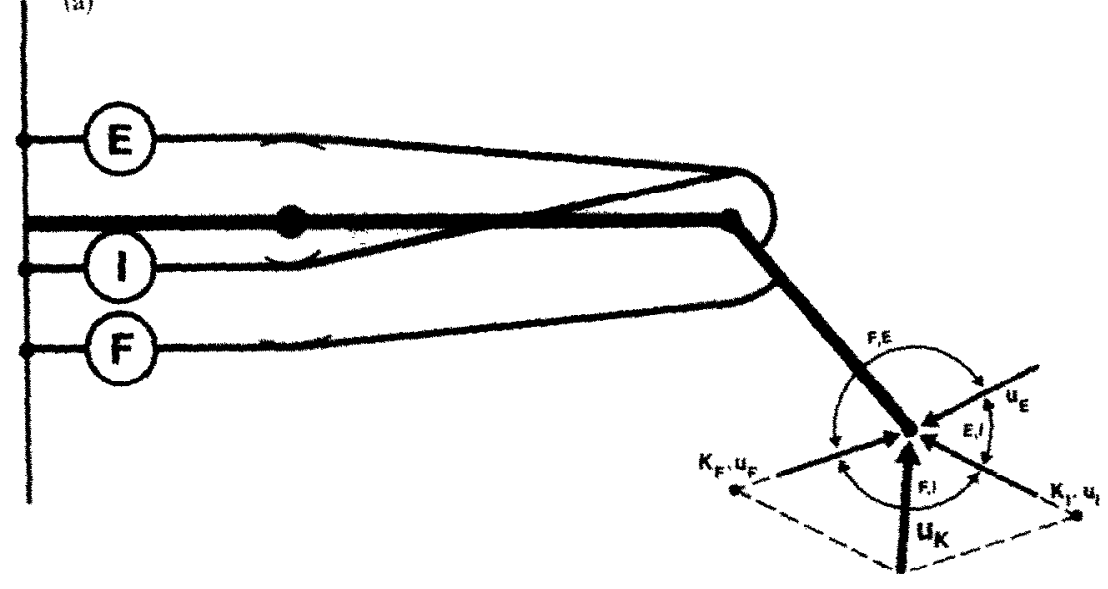

(b)

(E)

(1)

$F$

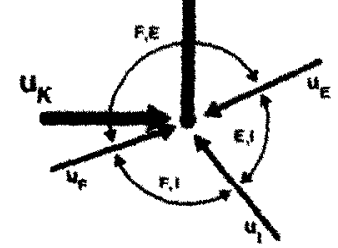

(c)

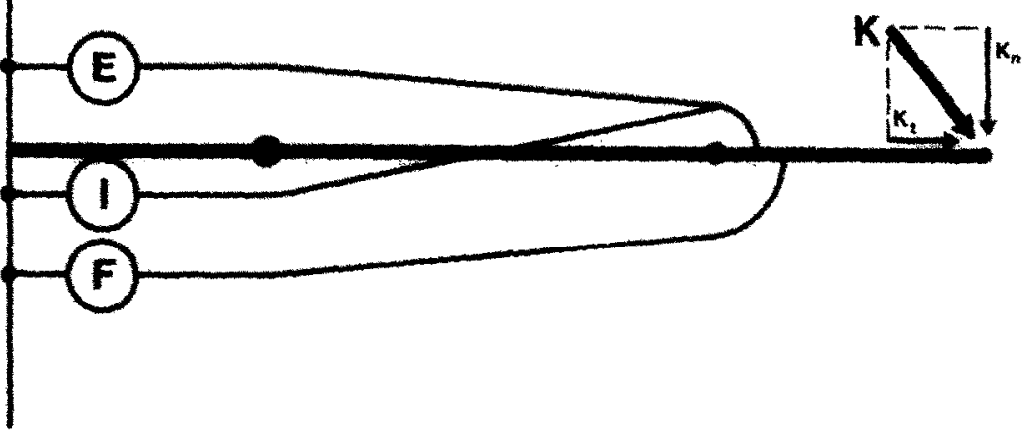

Fig. 3. (a) Laad vector diagram of the proximal bi-articular chain of the human finger. $K_{F}, K_{1}$ : moduli of the decomposition of the load on the unit load vectors $u_{F}, u_{i}$ of the motors $F$ and $l .(F, E),(E, I),(F, E)$ : the load ranges which can be balanced by these two motors alone. (b) Load $u_{K}$ as with carrying a bag with a bi-articular finger $\left(\theta_{2}=90^{\circ}, \phi=90^{\circ}\right)$. This load is within the $(F, E)$-load range. (c) The extension load at the finger tip of the extended finger can be decomposed into a tangential load $\mathbf{K}_{t}$ with zero moment arms $\left(\mathbf{V}_{1}=0\right)$, and a normal load $\mathbf{K}_{\mathrm{n}}$ with moment arms $\mathbf{V}_{\mathrm{n}}=\left(L_{1}+L_{2}, L_{2}\right)$ 
loaded and in the unloaded chain. It is independent of the position of the chain to the degree that the moment arms of the motors are independent of the joint position. The position of the chain is only reflected in the moment arm vector of the load.

(ii) The load vector diagram [expression (10)] shows in any position of the chain which motors balance a given load, but it does not allow to graphically derive the motor forces themselves.

\section{THE CONDITIONS FOR UNLOADED EQUILIBRIUM, LOADABILITY AND CONTROLLABILTTY OF THE BI-ARTICULAR CHAIN}

\section{The conditions for unloaded equilibrium}

The equilibrium of the unloaded chain is feasible only when the motor forces in expression (2) (with $K=0$ ) are non-negative and finite. From expressions (6a) it follows that the motor forces are strictly positive and finite when

$$
\begin{aligned}
& {\left[\mathbf{R}_{I} \times \mathbf{R}_{F}\right] \cdot\left[\mathbf{R}_{E} \times \mathbf{R}_{I}\right]>0,} \\
& {\left[\mathbf{R}_{F} \times \mathbf{R}_{E}\right] \cdot\left[\mathbf{R}_{E} \times \mathbf{R}_{I}\right]>0}
\end{aligned}
$$

(in Appendix B, equivalent conditions are derived for the load vector diagram). Conditions (11) can be interpreted in different ways from the vector diagram of Fig. 2(a).

(i) The left-hand members of expressions (6a) are the coefficients of the projections of the vector $-\mathbf{R}_{F}$ on the vector $\mathbf{R}_{E}$ and $\mathbf{R}_{I}$, as defined by expression (5). Conditions (11) state that these coefficients must be positive, i.e. that the vector $-\mathbf{R}_{F}$ must be a positive sum of $\mathbf{R}_{E}$ and $\mathbf{R}_{l}$. The conditions are symmetric: when the negative of one moment arm vector is expressible as a positive sum of the other two moment arm vectors, any of the three moment arm vectors is expressible as a positive sum of the other two moment arm vectors.

(ii) Each vector product term $\left[\mathbf{R}_{i} \times \mathbf{R}_{j}\right]$ is proportional to the sinus of the angle $\beta_{i j}$, measured anticlockwise between the vectors $\left\langle\mathbf{R}_{i}, \mathbf{R}_{j}\right\rangle$. As such, conditions (11) express that the equilibrium of the three-tendon chain is feasible when the sinus functions of the cyclic angles $\beta_{F I}, \beta_{I E}, \beta_{E F}$ between respectively $\left\langle\mathbf{R}_{F}, \mathbf{R}_{I}\right\rangle$, $\left\langle\mathbf{R}_{I}, \mathbf{R}_{E}\right\rangle,\left\langle\mathbf{R}_{E}, \mathbf{R}_{F}\right\rangle$ are all of the same sign, meaning that either $0<\beta_{F I}, \beta_{I E}, \quad \beta_{E F}<\pi$ or $\pi<\beta_{F I}, \beta_{I E}$, $\beta_{E F}<2 \pi$.

(iii) An interpretation in Landsmeer's model is given in Appendix $C$.

Mathematically, equilibrium may also be feasible with one or both conditions (11) equal to zero. This is explored in the next section. Note that a condition (11) is zero only when two moment arm vectors are collinear, i.e. $\left[\mathbf{R}_{i} \times \mathbf{R}_{j}\right]=0$ if and only if

$$
\mathbf{R}_{i}=\alpha \cdot \mathbf{R}_{j}
$$

Exact antagonism, well-loadable and well-controllable bi-articular chains

Presently, the mono-articular concept of muscular 'antagonism' is generalised for multi-articular motors, and the concepts of loadability and controllability of the multi-articular chain are defined.

(D1) Two motors $M_{i}, M_{j}$ with collinear moment arm vectors of opposite directions $[\alpha<0$ in expression (12)] are further called exact antagonists. Conversely, two motors with collinear moment arms of the same direction $(\alpha>0)$ are called exact agonists. When the moment arm vectors $\mathbf{R}_{i}, \mathbf{R}_{j}$ of two motors are collinear and opposite, their load vectors $\mathbf{u}_{i}, \mathbf{u}_{j}$ are also collinear and opposite. Contrary to the mono-articular antagonism, the concept of bi-articular exact antagonism is purely mathematic. In reality, moment arms change, however slightly, with joint position and motor force, which means that in the real bi-articular chain condition (12) cannot be consistently satisfied.

(D2) A chain is loadable when it can balance any unit load $u_{K}$ with finite motor forces $\left(M_{i}<\infty\right)$. When the chain is loadable, it is also controllable, since it can balance any perturbations of its equilibrium with finite motor forces.

(D3) The bi-articular chain is well loadable when it can withstand any unit load $\mathbf{u}_{K}$ with 'reasonable' motor forces $\left(M_{i} \ll \infty\right)$. When the chain is well loadable, it is also well controllable.

The conditions for loadability and controllability of the three-tendon bi-articular chain

The concept of exact antagonism allows formulatation of the conditions for equilibrium, loadability and controllability in physical terms.

(i) Two not exact antagonistic motors cannot balance the unloaded bi-articular chain. They cannot sustain any load outside their load range, which is less than $180^{\circ}$. The first statement follows from the fact that their moment arm vectors cannot balance out [in the generic expression (3)]. The second can be immediately verified from a load vector diagram [Fig. 3(a)].

(ii) Two exact antagonists can mathematically balance the unloaded bi-articular chain. However, they cannot sustain any load not collinear with their load vectors, and therefore cannot control the chain. Two exact antagonists $M_{i}, M_{j}$ can keep the unloaded (massless) bi-articular chain in equilibrium [substitute expression (12) in the generic expression (3) with $K=0$ ]. The force in a third motor must then be zero, except when its moment arm vector is also collinear with those of the exact antagonists. In the loaded two-tendon chain, the forces in exact antagonists with load vectors not collinear with the external load vector are infinite $\left(M_{i}, M_{j}=\infty\right)$ ) the vector product of the collinear moment arm vectors of the exact antagonists is zero $\left(\left[\mathbf{R}_{i} \times \mathbf{R}_{j}\right]=\left[-\alpha \cdot \mathbf{R}_{j} \times \mathbf{R}_{j}\right]=0\right)$ in expression (6b), while the product of these vectors with the moment arm vector of the load is not $\left(\left[\mathbf{R}_{i} \times \mathbf{V}_{\mathbf{K}}\right]=\left[-\alpha \cdot \mathbf{R}_{j} \times \mathbf{V}_{\mathbf{K}}\right] \neq 0\right)$.

(iii) Three motors satisfying expression (11) suffice for complete control of the bi-articular chain, in both the loaded and unloaded situation. When expressions (11) are satisfied, no motor pair is exact antagonistic, and the three moment arm vectors can balance out with positive forces. The moment arm vector of any load can then be 
written as a unique finite non-negative sum of two of the three moment arm vectors [Fig. 2(b)], meaning that the chain is fully loadable and controllable.

(iv) The bi-articular chain is well loadable, and well controllable, when conditions (11) are satisfied, and no motor pair is too antagonistic. When two motors approach exact antagonism, conditions (11) remaining satisfied, their forces required to balance loads in their common load range become very large, as $\left[\mathbf{R}_{i} \times \mathbf{R}_{j}\right] \rightarrow 0$ in expression (6b). Therefore, by definition, the loadability and the controllability of the chain decrease. A similar argument is as follows. In the unloaded chain, with two quasi-exact antagonists, the equilibrium force in the third motor is quasi-zero, meaning that the ratio of the forces in the quasi-exact antagonists and the force in the third motor is quasi-infinite. Since motors are physiologically limited in their (control of) force, such disbalances in the motor forces do not allow good control. Note that according to the above definitions, the controllability and loadability are optimal when the moment arm vectors of the motors are at $120^{\circ}$-i.e. with all motors of equal degree of antagonism.

\section{PROPERTIES AND APPLICATIONS}

Parameter analysis: the effects of changes in the moment arm vectors on motor force, loadability and controllability

The vector diagrams allow easy validation of the effects of changes in the motor moment arms on the motor forces, the feasibility of equilibrium, and the controllability and loadability of the bi-articular chain. Changes in moment arm vectors can be: (i) in the size of the moment arm vector, and (ii) and the direction of the moment arm vector. It holds that:

(i) A change in the size of the moment arm vector of a motor merely changes the effectiveness of this motor to the same proportion, and does not change the equilibrium forces in the other motors.

This follows from:

$$
\begin{aligned}
& \sum_{i} F_{i} \cdot \mathbf{R}_{i}+F_{j} \cdot \mathbf{R}_{j}=-K \cdot \mathbf{V}_{K} \\
& \quad \Rightarrow \sum_{i} F_{i} \cdot \mathbf{R}_{i}+\left(\frac{F_{j}}{\alpha}\right) \cdot\left(\alpha \cdot \mathbf{R}_{j}\right)=-K \cdot \mathbf{V}_{K},
\end{aligned}
$$

which shows that changing a moment arm vector $\mathbf{R}_{\boldsymbol{j}}$ by a factor $\alpha>0$ changes the corresponding motor force by a factor $1 / \alpha$, while leaving all other forces unchanged. In the unloaded case $(K=0)$, the change of effectivity is with respect to the other motor forces:

$$
\begin{aligned}
& \sum_{i} F_{i} \cdot \mathbf{R}_{i}+F_{j} \cdot \mathbf{R}_{j}=\mathbf{0} \\
& \quad \Rightarrow \sum_{i}\left(\alpha \cdot F_{i}\right) \cdot \mathbf{R}_{i}+F_{j} \cdot\left(\alpha \cdot \mathbf{R}_{j}\right)=\mathbf{0},
\end{aligned}
$$

which means that the increase of the moment arm vector $\mathbf{R}_{j}$ of a motor is equivalent with a decrease in the relative effectivity of all other motors. (ii) A change in the direction of a moment arm vector changes the relative forces in other motors, the load ranges, the loadability and the controllability. A change in the direction of the moment arm vector of a motor also changes the direction of its load vector in the load vector diagram, which means that the load ranges which this motor together with the other motors can sustain also change. Consequently, loads in the original load range of this motor will be balanced by different motors, or by the same motors with different forces, or cannot be balanced at all. Similarly, in the unloaded chain the variation of a moment arm vector direction changes the equilibrium forces of the motors relative to each other [expression (6a) ], and, most critically, may cause the violation of the positivity conditions (11) on the motor forces, resulting in an unbalanceable chain.

To summarise, the feasibility of unloaded equilibrium, the loadability and the controllability of the chain depend essentially on the relative directions of the moment arm vectors of the motors, while the effectiveness of the force-torque relationship of the individual motor depends on the moment arm vector size.

The moment arm vector and load vector diagrams of the bi-articular chain with extended second joint $\left(\theta_{2}=0\right)$

(i) The moment arm vector diagram. In the chain with an extended second joint $\left(\theta_{2}=0\right)$ [Fig. 3(c)], any load vector $K$ can be decomposed in a tangential component $\mathbf{K}_{\mathbf{t}}$ (collinear with the phalanges) and a normal component $K_{n}$ (perpendicular to the phalanges):

$$
\mathbf{K}=\mathbf{K}_{\mathbf{t}}+\mathbf{K}_{\mathrm{n}}=K \cdot|\cos \phi| \cdot \mathbf{u}_{\mathbf{t}}+K \cdot|\sin \phi| \cdot \mathbf{u}_{\mathrm{n}} \cdot
$$

The moment arm vectors of $u_{t}$ and $u_{n}$ are $V_{t}$ and $V_{n}$, respectively. The torque resulting from the tangential component $u_{t}$ is zero in both joints, since $V_{t}=0$. For all laods applied at the same point, the moment arms of the normal component $u_{n}$ are of equal length, while their sign depends only on whether the load is applied on the flexion (f) or the extension (e) side of the chain. In other words,

$$
\mathbf{V}_{\mathrm{n}}^{\mathrm{e}}=-\mathbf{V}_{\mathrm{n}}^{\mathrm{f}}, \quad\left\|\mathbf{V}_{\mathrm{n}}\right\|=\text { constant }
$$

From this it follows that in the extended chain all loads can be balanced by only two motor pairs, with one pair balancing all flexion loads and the other balancing all extension loads. The forces in these motor pairs are of a constant ratio, independent of the load direction, as from expression (6b) it follows that

$$
\begin{aligned}
\left(\frac{M_{i}}{M_{j}}\right)^{(e \text { or } f)} & =\frac{\left[\mathbf{V}_{K}^{(e \text { or } f)} \times \mathbf{R}_{j}\right]}{\left[\mathbf{R}_{i} \times \mathbf{V}_{K}^{(e \text { or } f)}\right]} \\
& =\frac{\left[\mathbf{V}_{\mathrm{n}}^{(\mathrm{e} \text { or } \mathrm{f})} \times \mathbf{R}_{j}\right]}{\left[\mathbf{R}_{i} \times \mathbf{V}_{\mathrm{n}}^{(\mathrm{e} \text { or } f)}\right]}=\mathrm{constant}
\end{aligned}
$$

since all vectors $V_{n}^{e}, V_{n}^{f}, R_{i}, R_{j}$ are independent of the load direction. In other words, whereas in the flexed chain different load directions applied at the same point are balanced by different motor pairs or by the same motor pair with different forces, in the extended chain the 
relative loading of the motors is constant for all fiexion or extension loads.

(ii) The load vector diagram. With extended second joint $\left(\theta_{2}=0\right)$, the load vector diagram is undefined. When $\theta_{2} \rightarrow 0$, the load vectors $\mathbf{u}_{M}$ of all motors $M$ become collinear with the joint axes [Fig. 4(a)]. Since their moment arms are then zero $\left[\mathbf{V}_{M}=0\right.$, and $\alpha_{M K}=0$ in expression (7)], they cannot balance any motor force anymore. Therefore, for the extended chain the load ranges of the motors cannot be straightforwardly deter- mined in the load vector diagram. Graphically, however, this problem can be circumvented by taking the limit of the load ranges for $\theta_{2} \rightarrow 0$. For instance, in Fig. $4(\mathrm{a})$ it is clear that when the second joint extends, the $(F, E)$-load range converges to the $180^{\circ}$ range of all extension loads; the $(F, I)$-load range converges to the $180^{\circ}$ range of all flexion loads; and the ( $E, I)$-load range converges to zero. From the corresponding moment arm vector diagram of Fig. 2(d) it can be verified that the motor pair $(F, E)$ indeed balances the unique moment arm vector $V_{n}^{e}$ of all
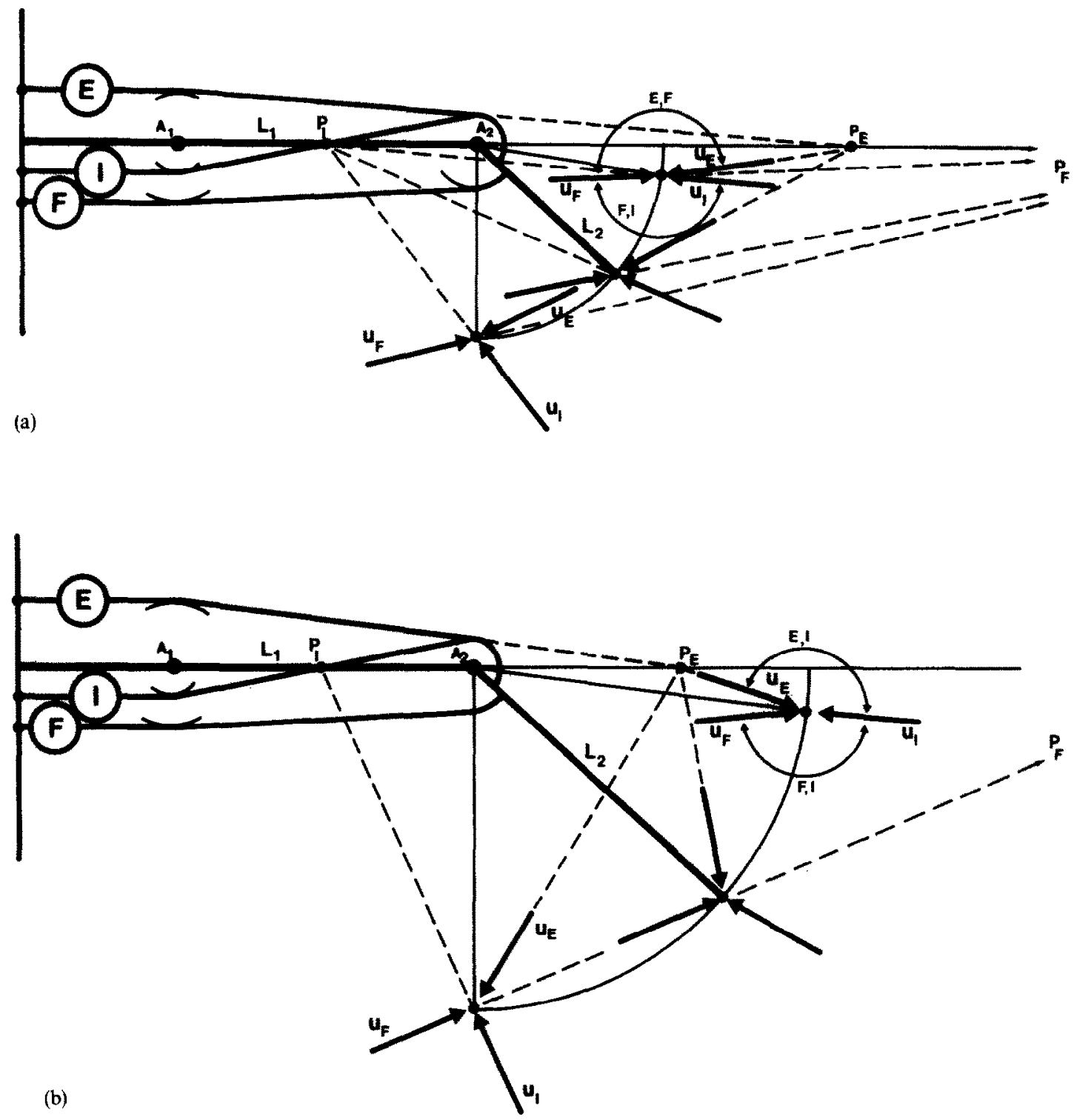

Fig, 4. (a) Load vector diagram of the extending proximal bi-articular chain of the human finger. Three PIP positions $\left(\theta_{2}=90,45,10^{\circ}\right)$ are drawn to demonstrate the changes in the load vector directions with PIP position. With extending PIP joint $\left(\theta_{2} \rightarrow 0\right)$, the $(F, E)$-load range increases to the $180^{\circ}$ range comprising all extension loads, the $(F, I)$-load range converges to the $180^{\circ}$ load range comprising all flexion loads, and the $(E, I)$-load range conveges to zero. (b) Same as Fig. $4(\mathrm{a})$, but with an elongated second phalanx. With extending PIP joint $\left(\theta_{2} \rightarrow 0\right)$, the $(E, I)$-load range converges to all extension loads, the $(F, I)$-load range converges to all flexion loads, and the $(F, E)$-load range converges to zero. 
extension loads, and the motor pair $(F, I)$ balances the unique moment arm vector $\mathbf{V}_{\mathbf{n}}^{\mathrm{f}}$ of all flexion loads.

The controllability and loadability of the proximal three-tendon bi-articular chain of the human finger

The proximal bi-articular chain of the human finger with the superficial flexor $(F)$, extensor $(E)$ and interosseus $(I)$ is given in Fig. 3(a), and the moment arm vector diagram in Fig. 2(a) (from the values of Table 1). From the above it follows that the feasibility of equilibrium, the loadability and the controllability are determined by the two most antagonistic motors. These are the extensor and flexor, which are almost exact antagonists. From this fact the following conclusions can be formulated:

(i) The proximal three-tendon bi-articular chain of the human finger is weak for loads in the load range of the extensor and flexor. In other words, the forces in extensor and flexor required to sustain unit loads in their load range will be high.

(ii) The controllability of the chain is not very good. This follows by definition from (i).

(iii) The functioning of the chain is not 'safe' with respect to small changes in the moment arms of extensor or flexor.

From expression (C3, Appendix C) or Fig. 2(c) it can be verified that the changes in the moment arms which result in unfeasible equilibrium [corresponding to the dashed vectors $\mathbf{R}_{E}^{*}$ or $\mathbf{R}_{F}^{*}$ in Fig. 2(c)] are relatively small. For instance, for changes in the moment arms at the MCP joint, equilibrium is unfeasible when:

$$
\begin{gathered}
\left|r_{E 1}\right|<\frac{\left|r_{F 1}\right|}{\left|r_{F 2}\right|} \cdot\left|r_{E 2}\right| \approx 7.2 \mathrm{~mm} \text { or } \\
\left|r_{F 1}\right|>\frac{\left|r_{E 1}\right|}{\left|r_{E 2}\right|} \cdot\left|r_{F 2}\right| \approx 16.2 \mathrm{~mm},
\end{gathered}
$$

which is a change of less than $2 \mathrm{~mm}$ and little more than $3 \mathrm{~mm}$ for the extensor and flexor, respectively. Clinically, such changes would correspond to extensor subluxation $\left(r_{E 1} \downarrow\right)$ or rupture of the flexor pulleys $\left(r_{F 1} \uparrow\right)$ at the MCP. Figure 2(c) also shows that simultaneous changes of only about $1 \mathrm{~mm}$ in the extensor or flexor moment arms, e.g. along the vectors $\Delta \mathbf{R}_{E}$ or $\Delta \mathbf{R}_{F}$, may result in an unbalanceable chain. Notice that such changes, especially in the extensor (subluxation of the tendon at the

Table 1. Moment arms of the motors of the proximal bi-articular chain of the human finger (in $\mathrm{mm}$ )

\begin{tabular}{lccrr}
\hline & \multicolumn{2}{c}{ Moment arm } & & \multicolumn{2}{c}{ Value } \\
\cline { 2 - 3 } \cline { 5 - 5 } Motor & MCP & PIP & MCP & PIP \\
\hline$F$ & $r_{E 1}$ & $r_{F 2}$ & -13 & -9 \\
$E$ & $r_{E 1}$ & $r_{E 2}$ & 9 & 5 \\
$I$ & $r_{I 1}$ & $r_{I 2}$ & -5 & 5 \\
Length first and second phalanx: 52,30 & &
\end{tabular}

From Spoor (1983).
MCP in combination with (dorsal) swelling of the PIP joint), may well be caused by rheumatoid arthritis.

(iv) Loads as may occur when carrying a bag, i.e. with $\phi=90^{\circ}$ and $\theta_{2}=90^{\circ} \quad[$ Fig. 3(b)], are within the flexor-extensor load range, and therefore cannot be well sustained. The reader may notice that this is well against intuition.

(v) The most efficient load range is of the flexor and interosseus [Fig. 3(a)]. This follows immediately from the fact that the angle between $\mathbf{R}_{I}$ and $\mathbf{R}_{F}$ is the smallest of all angles of the moment arm vectors. Note that this load range comprises the pinch grip loads.

To summarise, the proximal three-tendon bi-articular chain of the finger in itself cannot be considered as an optimal design: it cannot well sustain all physiologically occurring load situations, and is not especially adapted for good control. This indicates that in the real threearticular finger the other motors (deep flexor, lumbrical) will have to substantially improve upon the general load bearing capacity and the controllability.

\section{The forces in the extended proximal three-tendon} bi-articular chain of the human finger

In the above it is shown that with extended second joint $\left(\theta_{2}=0\right)$, all loads of flexion and extension, respectively, can be balanced by motor pairs with forces in a constant ratio. The question presently investigated is which motors and with what forces sustain these loads in the proximal bi-articular chain of the human finger. The parametric exploration of this question will illustrate the close relationship between geometry and function in the bi-articular chain, and the peculiar weakness for extension loads in the proximal bi-articular chain of the human finger.

The moment arm vector diagram of Fig. 2(d) or the load vector diagram of Fig. 4(a) show that a load applied at the flexion side to the tip of the extended chain is balanced by the flexor and the interosseus, while a load applied at the extension side is balanced by the extensor and the flexor (!). The latter fact is somewhat against intuition. Indeed, the extensors of the two joints are the extensor (MCP, PIP) and the interosseus (PIP). Therefore it could be expected that the extensor and the interosseus would balance extension loads. This may be compared with Fig. 4(b), where a bi-articular chain with a longer distal phalanx is considered. In this chain extension loads with $\theta_{2}=0$ are indeed balanced by the extensor and the interosseus [the $(E, I)$-load range converges to the hemisphere of the extension loads]. The only difference between Fig. 4(a) and (b) is the length of the distal phalanx, which determines the direction $v_{K 2} / v_{K 1}=L_{2} /\left(L_{1}+L_{2}\right)$ of the moment arm vector $-\mathbf{V}_{\mathrm{n}}^{\mathrm{e}}$ in the moment arm vector diagram of Fig. 2(d). From this diagram it is clear that:

(i) when $\left[-V_{n}^{e} \times R_{E}\right]>0$, extension load is balanced by the extensor and flexor;

(ii) when $\left[-V_{n}^{e} \times R_{F}\right]<0$, extension load is balanced by the extensor and interosseus;

(iii) when $\left[-V_{n}^{e} \times R_{E}\right]=0$, extension load is balanced by the extensor alone; 
(iv) when $\left[\mathbf{R}_{F} \times-\mathbf{V}_{\mathrm{n}}^{\mathrm{f}}\right]>0$, flexion load is balanced by the flexor and the extensor.

With extension loads at the end of the second phalanx of the proximal bi-articular chain of the human finger [case (i) of the above] [Fig. 4(a)], the extensor force is quite large, while the flexor force is about a third of the extensor force [Fig. 2(d)], even if $-V_{n}^{e}$ is almost collinear with the extensor moment arm vector $\mathbf{R}_{E}$. When the load is applied closer to the second joint (i.e. when $L_{2}$ decreases), the vector $-V_{n}^{e}$ is shifted towards the middle of the $(F, E)$-load range [Fig. 2(d)], and the extensor and flexor forces still increase considerably. This weakness for extension loads in the proximal finger can be self-verified by applying an extension load close and distal to the PIP joint of ones own fingers (hereby the PIP joint must be kept slightly flexed, so that the palmar joint ligaments, i.e. the volar plate, remain slack). One will experience that even small loads are difficult to balance, and that the PIP will tend to extend completely. Also it can be observed that when the PIP is fully extended and the volar plate is taut, much greater extension loads can be sustained. The taut volar plate effectively functions as a mono-articular flexor of the PIP, with a moment arm vector $\mathbf{R}_{\mathrm{YP}}$ as in Fig. 4(d). This 'flexor' force of the volar plate may completely substitute the superfical flexor, and creates a more efficient load range in which the extensor requires less than half of the force with a slack volar plate.

\section{DISCUSSION}

In the present paper a model is introduced which visualises the relationship between morphology and function in the bi-articular chain, and which allows us to graphically analyse the motor forces in the unloaded and loaded chain, the load ranges of the motors, the ranges of preferential loading (i.e. the loads which can be sustained with minimal force), the feasibility of equilibrium and the condition of control. The present model generalises the results of Landsmeer (1955) and Spoor and Landsmeer (1976), who investigated the control of the unloaded bi-articular chain in morphological terms. The graphic representation of the present model is possible because the kinematics and statics of the massless frictionless bi-articular chain depend entirely upon its geometry, i.e. the lengths of the moment arms of motors and loads.

The present model may be valuably applied to investigate the functioning of bi-articular chains in which the motor forces and loads are large as compared to the masses of the phalanges, as is the case in fingers or toes, or to investigate the statics of chains in the horizontal plane, i.e. with the gravity forces parallel to the joint axes. Hereby the moment arms may well be functions of the joint position, since at any given position of the chain they have a fixed value, and the model is valid. In the text the model is applied to investigate the properties of the proximal three-tendon bi-articular chain of the human finger. It clearly emerges that this chain is especially designed to sustain (certain) 'flexion' loads, that it is structurally weak for extension loads, and that its controllability is not optimal. This indicates that the third joint and the other finger motors (deep flexor and lumbrical) play an important role in improving finger control.

The two-dimensional vector diagrams here presented cannot be extended to the $n$-articular chain, with $n>2$. In the vector diagrams the moment arms of each independent joint axis are represented at an independent Cartesian axis, which means that an $n$-articular chain requires a $n$-dimensional vector representation. Moreover, for the $n$-articular chain (with $n>2$ ), the load vectors of the motors generally do not exist, since their load line must be tangential to all circles, centred at the joint axes, and of which the radii are in a fixed proportion [hy the constant $\alpha_{M K}$ in expression (7)] to the moment arms of their motors at these joints. Generally, this condition is not satisfied for $n>2$.

\section{CONCLUSION}

A model is presented which allows to graphically investigate the equilibrium conditions, the loadability and the controllability of the bi-articular chain. To allow a concise formulation of the results, the concept of exact antagonism of bi- (or multi-) articular motors is introduced. It is shown that:

(i) Two not exact antagonistic motors cannot keep the chain in equilibrium, and cannot sustain all loads.

(ii) Two exact antagonists can mathematically balance the chain, but cannot control it.

(iii) The bi-articular chain with three motors is fully controllable and loadable when no two motors are exact antagonists, and no moment arm vector can be written as a positive sum of the others. The bi-articular chain with $n$ motors is fully loadable and controllable when a motor triplet exists which satisfies this criterion.

(iv) All loads can be sustained by maximally two motors.

(v) When two motors approach exact antagonism, the three-motor chain cannot be well controlled. Moreover, the common load range of these quasi-exact antagonists is structurally weak.

(vi) With extended second joint, all loads of extension can be balanced by the same motor pair with forces in a fixed ratio. Similarly, a (different) motor pair will balance all flexion loads with fixed force ratio.

With respect to the proximal three-tendon bi-articular chain of the human finger, it holds that the loadability and controllability of the chain of the proximal finger is critically determined by the pronounced antagonism of the extensor and superficial flexor. The other motor pairs are not at all antagonistic. The chain is structurally weak in the extensor-flexor load range, and is not well conditioned for good control. Moreover, relatively small changes in the moment arms of extensor and/or flexor may result in an uncontrollable chain.

Acknowledgements - The author cordially thanks Prof. Emer, J. M. F. Landsmeer, Prof. J. J. Kalker, Dr C. W. Spoor, Prof. C. J. Snijders, Prof. J. C. Van der Meulen and Dr S. E. R. Hovius for their valuable contributions. 


\section{REFERENCES}

Landsmeer, J. M. F. (1955) Anatomical and functional investigations on the articulation of the human fingers. Acta Anat. 25 (Suppl. 24), 1-69.

Leijnse, J. N. A. L. and Kalker, J. J. (1995) A two dimensional kinematic model of the lumbrical in the human finger. $J$. Biomechanics 28, 237-249.

Spoor, C. W. (1983) Balancing a force on the fingertip of a two dimensional finger model without intrinsic muscles. $J$. Biomechanics 16, 497-504.

Spoor, C. W. and Landsmeer, J. M. F. (1976) Analysis of the zigzag movement of the human finger under influence of the extensor digitorum tendon and the deep flexor tendon. $J$. Biomechanics 9, 561-566.

\section{APPENDIX A}

\section{MOTOR FORCES IN TERMS OF MOMENT ARM VECTORS}

Assume that no moment arm vectors are collinear. Expressions (6) are obtained by taking the vector product of the motor moment arms and the vector equation (3). For instance, the vector product of the extensor moment arm vector $\mathbf{R}_{E}$ with expression (3) is

$$
\begin{aligned}
E \cdot\left[\mathbf{R}_{E} \times \mathbf{R}_{E}\right]+I \cdot\left[\mathbf{R}_{E} \times \mathbf{R}_{I}\right]+F \cdot & {\left[\mathbf{R}_{E} \times \mathbf{R}_{F}\right] } \\
& =-K \cdot\left[\mathbf{R}_{E} \times \mathbf{V}_{K}\right] .
\end{aligned}
$$

It holds that $\mathbf{R}_{E} \times \mathbf{R}_{E}=\mathbf{0}$. Therefore, for the unloaded case $(K=0)$, the relationship $I / F$ of expression (6) is obtained:

$$
\frac{I}{F}=\frac{-\left[\mathbf{R}_{E} \times \mathbf{R}_{F}\right]}{\left[\mathbf{R}_{E} \times \mathbf{R}_{I}\right]}=\frac{\left[\mathbf{R}_{F} \times \mathbf{R}_{E}\right]}{\left[\mathbf{R}_{E} \times \mathbf{R}_{I}\right]} .
$$

The other forces can be derived similarly.

\section{APPENDIX B}

\section{CONDITIONS FOR EQUILIBRIUM IN THE LOAD VECTOR DIAGRAM}

The massless chain is in equilibrium with a motor and its load vector. Therefore, the superposition of all motors and their load vectors also results in a chain in equilibrium. This equilibrium is not disturbed when, for any motor, the motor force and its load vector are multiplied by the same positive number $K_{M}$. The vector sum of all load vectors is the effective load with which the chain with the given motor forces is in equilibrium. When this sum is zero (the load vectors, themselves not zero, balance out), an unloaded chain is obtained. From this it follows that the motors can keep the chain in equilibrium, when the vector sum of their load vectors can be put to zero:

$$
K_{F} \cdot \mathbf{u}_{F}+K_{I} \cdot \mathbf{u}_{I}+K_{E} \cdot \mathbf{u}_{E}=\mathbf{0}
$$

with $K_{F}, K_{I}, K_{E} \geqslant 0$, and nut all of them zero. This expression is similar to expression (3) and leads, similarly to expression (11), to the conditions that $\infty>K_{F}, K_{E}, K_{I}>0$ when

$$
\begin{aligned}
& {\left[\mathbf{u}_{I} \times \mathbf{u}_{F}\right]\left[\mathbf{u}_{E} \times \mathbf{u}_{I}\right]>0,} \\
& {\left[\mathbf{u}_{F} \times \mathbf{u}_{E}\right]\left[\mathbf{u}_{E} \times \mathbf{u}_{I}\right]>0 .}
\end{aligned}
$$

Conditions (B2) and (11) are consistent: expressions (B2) hold if and only if expressions (11) hold [except in the extended chain $\left(\theta_{2}=0\right)$ where the vectors $\mathbf{u}_{i}$ are undefined], while also the graphic interpretation is similar to expression (11).

APPENDIX $\mathrm{C}$

LANDSMEER CONDITIONS FOR EQULLIBRIUM OF THE PROXIMAL BI-ARTICULAR CHAIN OF THE HUMAN FINGER

With expression (1), conditions (11) can be written as

$$
\begin{aligned}
& {\left[r_{E I} \cdot r_{I 2}-r_{E 2}-r_{I 1}\right] \cdot\left[r_{I 1} \cdot r_{F 2}-r_{I 2} \cdot r_{F 1}\right]>0,} \\
& {\left[r_{E I} \cdot r_{I 2}-r_{E 2}-r_{I 1}\right] \cdot\left[r_{F 1} \cdot r_{E 2}-r_{F 2} \cdot r_{E 1}\right]>0 .}
\end{aligned}
$$

For the proximal bi-articular chain of the human finger it holds that $r_{E 1}, r_{E 2}, r_{I 2}>0$ and $r_{I 1}, r_{F 1}, r_{F 2}<0$ [see Table 1 or Fig. 1(a)]. With these values, the inequalities (C1) can be written as:

$$
\begin{array}{r}
{\left[\frac{\left|r_{I 2}\right|}{\left(-\left|r_{I 1}\right|\right)}-\frac{\left|r_{E 2}\right|}{\left|r_{E 1}\right|}\right] \cdot\left[\frac{\left(-\left|r_{F 2}\right|\right)}{\left(-\left|r_{F 1}\right|\right)}-\frac{\left|r_{I 2}\right|}{\left(-\left|r_{I 1}\right|\right)}\right]<0,} \\
{\left[\frac{\left|r_{I 2}\right|}{\left(-\left|r_{I 1}\right|\right)}-\frac{\left|r_{E 2}\right|}{\left|r_{E 1}\right|}\right] \cdot\left[\frac{\left|r_{E 2}\right|}{\left|r_{E 1}\right|}-\frac{\left(-\left|r_{F 2}\right|\right)}{\left(-r_{F 1} \mid\right)}\right]>0 .}
\end{array}
$$

Each term between square brackets is the difference of the directions $r_{M 2} / r_{M 1}$ of the moment arm vectors $\mathbf{R}_{M}$ of two motors, and therefore provides a measure for their degree of antag onism. For exact antagonists (or agonists) the term is zero. In expression (C2) two terms are 'safe', that is, not likely to change in sign with small variations in the ratios $r_{M_{2}} / r_{M_{1}}$. These are the $(E, I)$-term, which is the sum of two negative ratios, and the $(F, I)$-term, which is the sum of two positive ratios. These terms express that the $(F, I)$ and $(E, I)$ motor pairs are not at all antagonistic. However, the $(F, E)$-term is the sum of two ratios of different sign, and its sign may well change with small variations in the moment arm ratios. Therefore, this term (which expresses the antagonism of the $F$ and $E$ ) critically determines the feasibility of equilibrium of the chain. When the term is negative, the chain is controllable; when it is positive, the chain cannot be balanced. In Landsmeer (1955) and Spoor and Landsmeer (1976), the terms of $(F, I)$ and $(E, I)$ were not considered (they are not critical) and the controllability condition of the chain was derived in the form

$$
\frac{\left|r_{E 1}\right|}{\left|r_{F 1}\right|}-\frac{\left|r_{E_{2}}\right|}{\left|r_{F_{2}}\right|}>0
$$

